\title{
The effect of trial-to-trial feedback on the error-related negativity and its relationship with anxiety
}

\author{
Doreen M. Olvet and Greg HajCaK \\ Stony Brook University, Stony Brook, New York
}

\begin{abstract}
Individuals with anxiety disorders and related personality traits are characterized by increased error-related brain activity, as measured by the error-related negativity (ERN) in simple speeded response tasks. An absent, or opposite, relation between anxiety and the ERN has been reported in studies that employed reinforcement learning paradigms with trial-to-trial feedback. Understanding the effect of trial-to-trial feedback on the ERN may help clarify these results and can further elucidate the impact of feedback on performance monitoring. In the present study, 30 undergraduate participants performed two versions of the arrowhead version of the flanker task in counterbalanced order: one with trial-to-trial feedback and one without. The participants were slower and more accurate in the task with trial-to-trial feedback; however, the ERN was equivalent between the two tasks. Larger ERNs were related to higher trait anxiety, but only in the version without trial-to-trial feedback. These findings show that although trial-to-trial performance feedback impacts behavioral measures, it does not affect the ERN; moreover, the presence of trial-to-trial feedback moderates the relationship between the ERN and anxiety.
\end{abstract}

Neural measures of error processing have been featured in contemporary studies in developmental (Davies, Segalowitz, \& Gavin, 2004), cognitive (Gehring, Goss, Coles, Meyer, \& Donchin, 1993), clinical (Hajcak, Franklin, Foa, \& Simons, 2008), and social (Amodio, Jost, Master, \& Yee, 2007) psychology. In particular, these studies have focused on an event-related potential (ERP) known as the error-related negativity (ERN). The ERN presents as a negative deflection approximately $50 \mathrm{msec}$ following an erroneous response at frontal-central midline recording sites (Falkenstein, Hohnsbein, Hoormann, \& Blanke, 1991; Gehring et al., 1993). The neural source of the ERN appears to be the anterior cingulate cortex (Dehaene, Posner, \& Tucker, 1994; Holroyd, Dien, \& Coles, 1998; van Veen \& Carter, 2002), and it has been hypothesized that it represents the early detection of events that require increased cognitive control (Ridderinkhof, Ullsperger, Crone, \& Nieuwenhuis, 2004) - for instance, events that are worse than anticipated (Holroyd \& Coles, 2002) or when there is increased response conflict (Yeung, Cohen, \& Botvinick, 2004). The ERN is observed regardless of stimulus and response modalities (Bernstein, Scheffers, \& Coles, 1995; Holroyd et al., 1998; Van’t Ent \& Apkarian, 1999 ) and is similarly observed for both simple (Hajcak \& Foti, 2008; Hajcak \& Simons, 2008) and difficult (Band \& Kok, 2000; Compton et al., 2008; Mathalon et al., 2003; Themanson, Hillman, \& Curtin, 2006) tasks.

To elicit the ERN, studies have employed relatively simple speeded response tasks. For example, in versions of the flanker task, participants are instructed to respond to the identity or direction of a centrally presented stimulus, while ignoring the identity or direction of the surrounding stimuli. In an arrowhead version of the flanker task, participants have to respond to the direction of a central arrowhead on both compatible (e.g., $<<<<<$ ) and incompatible (e.g., $<<><<$ ) trials (Eriksen \& Eriksen, 1974). These tasks are fairly simple, and participants are instructed to respond both quickly and accurately. Although accuracy tends to be high, participants occasionally commit errors - presumably due to impulsive responding before stimulus processing has been fully completed (Rabbitt \& Vyas, 1981).

The ERN has been a useful tool for understanding abnormal error processing in certain clinical populations, such as individuals with anxiety disorders. Gehring, Himle, and Nisenson (2000) first demonstrated that individuals with obsessive-compulsive disorder (OCD) had larger ERNs than did healthy controls, and these data were taken to reflect exaggerated error signals that might underlie the cycle of obsessions and compulsions that characterizes patients with OCD. Although a number of researchers have subsequently replicated the finding that OCD and obsessive-compulsive traits are related to an increased ERN (Hajcak et al., 2008; Hajcak \& Simons, 2002; Johannes et al., 2001; Ruchsow et al., 2005; Santesso, Segalowitz, \& Schmidt, 2006), it appears that this effect is not specific to OCD. For example, some studies have shown larger ERNs among individuals who are worried 
(Hajcak, McDonald, \& Simons, 2003) and depressed (Chiu \& Deldin, 2007; Holmes \& Pizzagalli, 2008). Consistent with these results, an increased ERN has been related to the higher order construct of negative affect that might characterize both anxious and depressed individuals (Hajcak, McDonald, \& Simons, 2004; Luu, Collins, \& Tucker, 2000; Olvet \& Hajcak, 2008). Importantly, studies relating the ERN to anxiety and related personality traits have utilized simple speeded response tasks.

When more complex tasks (i.e., tasks that incorporate learning) have been employed to study error processing in anxiety disorders, results have been mixed, if not opposite. For instance, in a number of studies, a reinforcement learning task has been used to study the ERN, in which participants are provided with trial-to-trial feedback to learn the appropriate stimulus-response mappings (e.g., Holroyd \& Coles, 2002). Nieuwenhuis, Nielen, Mol, Hajcak, and Veltman (2005) used a reinforcement learning task and found that individuals with OCD had ERNs comparable to those of healthy controls. The authors suggested that the failure to find an increased ERN among OCD patients in this study may have been due to the presence of trial-to-trial feedback in the reinforcement learning task; that is, trial-to-trial feedback may reduce error-monitoring demands and, therefore, alter the relationship between the OCD and the ERN (Nieuwenhuis et al., 2005). Consistent with this possibility, a recent study examined the ERN among individuals with high and low OCD symptoms, using both a reinforcement learning and a simple flanker task; in this study, individuals reporting OCD symptoms had larger ERNs in the flanker task and smaller ERNs in the reinforcement learning task (Gründler, Cavanagh, Figueroa, Frank, \& Allen, 2009). Although the simple flanker task and the reinforcement learning task differ on several dimensions, these data are consistent with the possibility that the presence of trial-to-trial feedback itself influences the relationship between the ERN and individual differences in anxiety.

The implications of these findings extend beyond anxiety disorders and individual differences to more fundamental issues regarding performance monitoring: It is possible that trial-to-trial feedback may alter how errors are processed. For instance, it is possible that receiving performance feedback could increase emphasis on performance accuracy, which has been shown to increase the ERN in previous work (Gehring et al., 1993). However, it is also possible that internal and external performancemonitoring systems interact in a reciprocal fashion to optimize behavioral performance. That is, when presented with trial-to-trial performance feedback, participants may rely less on internal error monitoring, which would be reflected in a diminished ERN (Nieuwenhuis et al., 2005). The latter possibility is supported by studies that have demonstrated an inverse relationship between the ERN and a similar ERP response to negative feedback in reinforcement learning tasks: When individuals must rely more on external feedback, the ERN is decreased (Holroyd \& Coles, 2002; Nieuwenhuis et al., 2002). As with reinforcement learning tasks, some studies in which sim- ple speeded response tasks have been utilized have also included trial-to-trial feedback (i.e., Holmes \& Pizzagalli, 2008; Schrijvers et al., 2008; Schrijvers et al., 2009); however, in these studies, the feedback often conveys redundant information, because the participants are aware of their mistakes. Yet it is not known how the mere presence of performance feedback may impact the internal detection of errors.

To date, the effect of trial-to-trial feedback on the ERN has not been examined using a within-subjects design. In other words, it is not clear whether the presentation of extraneous trial-to-trial feedback itself serves as a manipulation of the ERN. To address this issue, performance and ERP measures were recorded while participants performed a speeded response task with and without trialto-trial performance feedback. The presence of trial-totrial feedback might induce more cautious responding, as indicated by increased accuracy and decreased reaction time. If this were the case, trial-to-trial feedback should be associated with a larger ERN, on the basis of data relating improved behavioral measures to a larger ERN (Gehring et al., 1993). Conversely, participants might reduce internal monitoring in the condition with trial-to-trial performance feedback, which should be associated with a smaller ERN (Holroyd \& Coles, 2002; Nieuwenhuis et al., 2002). In addition, the relationship between the ERN in both tasks and self-reported symptoms of anxiety was examined. On the basis of recent work suggesting that the presence of performance feedback may alter the relationship between the ERN and OCD symptoms (Gründler et al., 2009; Nieuwenhuis et al., 2005), it was expected that higher self-reported anxiety would relate to a larger ERN in the condition without feedback but a smaller ERN in the condition with feedback.

\section{METHOD}

\section{Participants and Measures}

Thirty undergraduates (19 male, 11 female) participated in the present study. Participants who made fewer than six errors in either task were excluded (Olvet \& Hajcak, 2009); thus, 1 participant was excluded, and the final sample consisted of 29 participants (11 female). For the behavioral data, 2 subjects were not included, due to a computer error. Informed consent was obtained from the participants prior to the experiment. This research was approved by the Stony Brook University Institutional Review Board. No participants discontinued their participation in the experiment once procedures had begun, and all the participants received course credit for their participation.

To assess the influence of symptoms of anxiety on error monitoring, the participants completed the short-form version of the Depression Anxiety Stress Scales (DASS-21; Lovibond \& Lovibond, 1995). The DASS - 21 is a 21-item self-report that is divided into three scales: depression, anxiety, and stress. There are 7 items for each scale, and the participants were instructed to respond to each item on the basis of how they had felt for the past week, using a 0-3 scale with $0=$ did not apply to me and $3=$ applied to me much or most of the time. The reliability and validity of the DASS-21 in both clinical and nonclinical samples has been previously established (Antony, Bieling, Cox, Enns, \& Swinson, 1998; Brown, Chorpita, Korotitsch, \& Barlow, 1997; Clara, Cox, \& Enns, 2001; Crawford \& Henry, 2003; Henry \& Crawford, 2005; Lovibond \& Lovibond, 1995). 


\section{Task and Materials}

The present tasks were administered on a Pentium D class computer, using Presentation software (Neurobehavioral Systems, Albany, CA) to control the presentation and timing of all the stimuli. The task was an arrow version of the flanker task (Eriksen \& Eriksen, 1974; Moser, Hajcak, \& Simons, 2005). On each trial, five horizontally aligned arrowheads were presented. Half of all trials were compatible $(<<<<<$ or $>>>>>$ ), and half were incompatible $(<<><<$ or $>><>>)$, and the order of compatible and incompatible trials was random. All the stimuli were presented for $200 \mathrm{msec}$, followed by an interval that varied randomly from 2,300 to $2,800 \mathrm{msec}$; this value reflected the interstimulus time between flanker offset and subsequent onset. In the condition with feedback, $500 \mathrm{msec}$ after the participants made their responses, they received feedback to indicate whether or not they had responded correctly. The feedback stimuli were presented for $500 \mathrm{msec}$ and consisted of a schematic frowning ( $(:$; negative feedback) or smiling ( $(-)$; positive feedback) face; these stimuli were not presented in the condition without feedback.

\section{Procedure}

Following a brief description of the experiment, electroencephalograph (EEG) sensors were attached, and the participant was given detailed task instructions. Each participant performed the flanker task both with and without trial-to-trial feedback, and the order of the task conditions was counterbalanced across participants. The participants were instructed to press the right mouse button if the center arrow was facing to the right and to press the left mouse button if the center arrow was facing to the left. For each condition, the participants performed a practice block containing 30 trials and were instructed to be both as accurate and as fast as possible. Each condition consisted of 11 blocks of 30 trials. To encourage both fast and accurate responding in both conditions, the participants received feedback based on their performance at the end of each block. If performance was $75 \%$ correct or lower, the message "Please try to be more accurate" was displayed; performance above $90 \%$ correct was followed by "Please try to respond faster"; otherwise, the message "You're doing a great job" was displayed. The only differences between the two task conditions were the trial-to-trial feedback and a longer intertrial interval in the feedback condition.

\section{Psychophysiological Recording, Data Reduction, and Analysis}

The continuous EEG activity was recorded using an ActiveTwo head cap and the ActiveTwo BioSemi system (BioSemi, Amsterdam). Recordings were taken from 64 scalp electrodes based on the 10/20 system, as well as 2 electrodes placed on the left and right mastoids. The electrooculogram (EOG) generated from blinks and eye movements was recorded from 4 facial electrodes: 2 approximately $1 \mathrm{~cm}$ above and below the participant's right eye (vertical EOG bipolar recording), 1 approximately $1 \mathrm{~cm}$ to the left of the left eye, and 1 approximately $1 \mathrm{~cm}$ to the right of the right eye (horizontal EOG bipolar recording). As per BioSemi's design, the ground electrode during acquisition was formed by the Common Mode Sense active electrode and the Driven Right Leg passive electrode. The EEG was sampled at $512 \mathrm{~Hz}$. All bioelectric signals were digitized on a laboratory microcomputer using ActiView software (BioSemi, Amsterdam).

Offline analysis was performed using Brain Vision Analyzer software (Brain Products, Gilohing, Germany). EEG data were rereferenced to the numeric mean of the mastoids and band-pass filtered with cutoffs of 0.1 and $30 \mathrm{~Hz}$. The EEG was segmented for each trial, beginning $400 \mathrm{msec}$ before each picture onset and continuing for $1,000 \mathrm{msec}$. The EEG was corrected for blinks and eye movements, using the method developed by Gratton, Coles, and Donchin (1983). Specific intervals for individual channels were rejected on each trial, using a semiautomated procedure, with physiological artifacts identified by the following criteria: a voltage step of more than $50.0 \mu \mathrm{V}$ between sample points, a voltage difference of $300.0 \mu \mathrm{V}$ within a trial, and a maximum voltage difference of less than $0.50 \mu \mathrm{V}$ within 100-msec intervals; all segments were also visually inspected for additional artifacts.

Response-locked average ERPs were computed for correct and error trials. The ERN and correct response negativity (CRN) were quantified on error and correct trials, respectively, as the average activity in a 0 - to $100-\mathrm{msec}$ window, relative to response onset at electrode site $\mathrm{FCz}$, where the ERN was maximal. The Pe was evaluated on error trials as the average activity from 200 to $400 \mathrm{msec}$ following response onset at electrode site $\mathrm{Pz}$. A 200-msec window prior to the response ( -400 to $-200 \mathrm{msec}$ ) served as the baseline.

In all cases, behavioral and ERP data were statistically evaluated using SPSS General Linear Model software (Version 16.0; SPSS Inc., Chicago). A trial (correct vs. incorrect) $\times$ condition (feedback vs. no feedback) ANOVA was used to detect differences between the two tasks, and paired $t$ tests were performed for follow-up post hoc comparisons. A paired-sample $t$ test was used to determine the effect of condition on the Pe. The Pearson correlation coefficient $(r)$ was used to examine the relationship between the ERN and Pe in the conditions with and without feedback, as well as the relationship between the ERN and Pe and the DASS-21 scores. Fisher's $z$ was used to examine differences between correlation coefficients.

\section{RESULTS}

\section{Behavioral Results}

Behavioral data are presented in Table 1. The participants committed fewer errors on the flanker task when they received trial-to-trial feedback $[M=26.48, S D=$ 11.88 , vs. $M=46.78, S D=21.85 ; t(28)=5.39, p<$ $.001]$. Number of errors was significantly correlated between the task conditions $[r(27)=.45, p<.05]$. Consistent with previous studies, the participants responded more quickly on error than on correct trials $[F(1,26)=$ 151.27, $p<.001]$, and they were faster in the condition without trial-to-trial feedback $[F(1,26)=8.33, p<.01]$; reaction time differences between correct and error trials did not vary between the conditions with and without

Table 1

Behavior and ERP Data

\begin{tabular}{|c|c|c|c|c|}
\hline & \multicolumn{2}{|c|}{$\begin{array}{l}\text { Without Trial-to- } \\
\text { Trial Feedback }\end{array}$} & \multicolumn{2}{|c|}{$\begin{array}{l}\text { With Trial-to- } \\
\text { Trial Feedback }\end{array}$} \\
\hline & $M$ & $S D$ & $M$ & $S D$ \\
\hline No. of errors & 46.78 & $21.85^{*}$ & 26.48 & $11.88^{*}$ \\
\hline Error trial reaction time (msec) & 331.28 & $65.61^{* *}$ & 351.64 & $40.92^{* *}$ \\
\hline Correct trial reaction time (msec) & 389.61 & $39.23^{* *}$ & 412.86 & $39.61^{* *}$ \\
\hline Error-related negativity $(\mu \mathrm{V})$ & 0.32 & 6.89 & 1.13 & 6.94 \\
\hline Correct response negativity $(\mu \mathrm{V})$ & 8.65 & 4.87 & 9.00 & 6.19 \\
\hline
\end{tabular}

${ }^{*} p<.001 .{ }^{* *}$ Both correct and error trial reaction times were significantly longer in the task with feedback $(p<.01)$. 
Without Trial-to-Trial Feedback

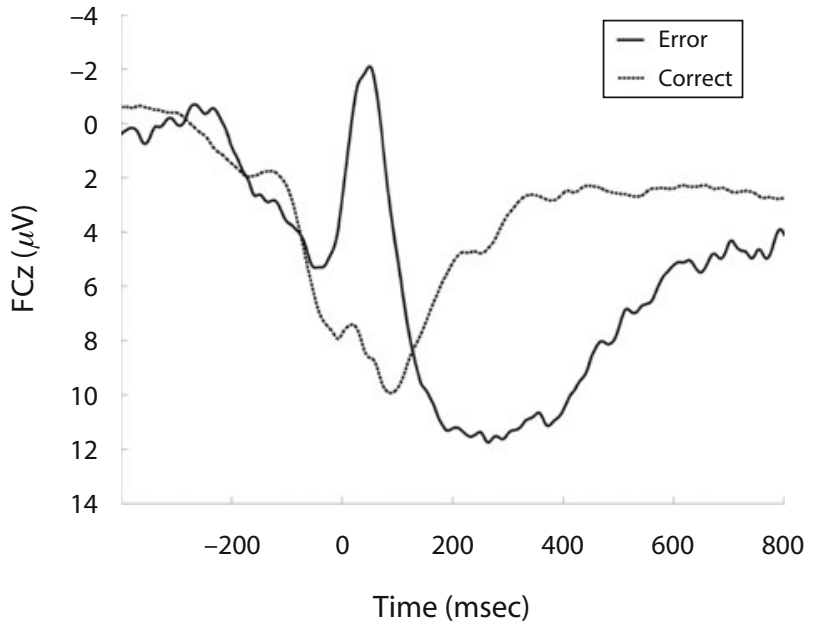

With Trial-to-Trial Feedback

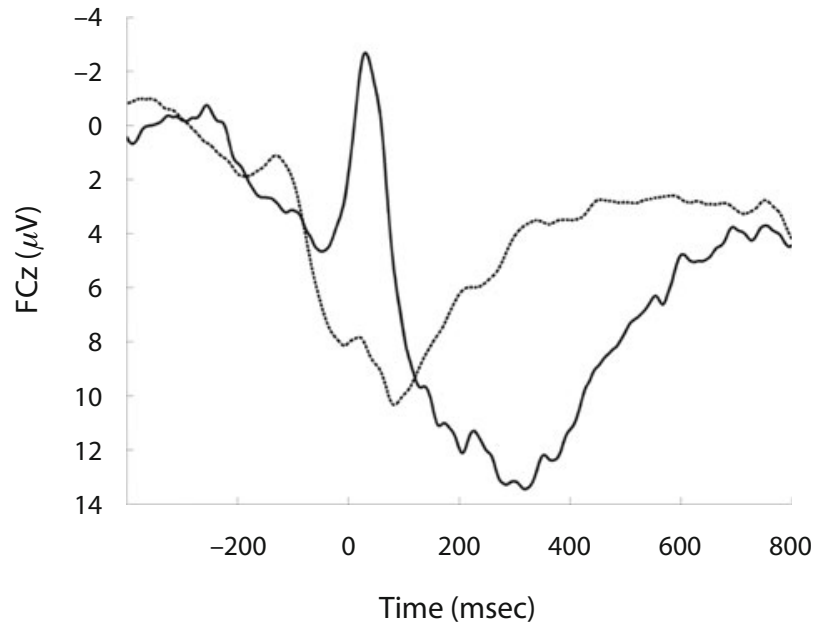

Figure 1. The response-locked event-related potentials for error and correct trials at FCz, where the ERN was maximal for the flanker task without feedback (left) and with feedback (right). Response onset occurred at 0 msec, and negative is plotted up.

feedback $[F(1,26)<1]$. Therefore, the participants were both slower and more accurate when they received trialto-trial feedback. In terms of speed-accuracy trade-off, the presence of trial-to-trial feedback was associated with more cautious performance.

\section{ERP Results}

Figure 1 presents response-locked ERPs from the flanker task without feedback (left) and with feedback (right), and the average ERP values are presented in Table 1. As was expected, the ERN was significantly more negative than the CRN $[F(1,28)=112.58, p<.001]$. However, the ERN did not differ between the tasks with and without feedback $[F(1,28)<1]$; the interaction between trial and condition also did not reach significance $[F(1,28)<1]$. The magnitudes of the ERN in the conditions with and without feedback were highly correlated $[r(27)=.72$, $p<.001]$; Figure 2 depicts the ERN in the condition without feedback as a function of the ERN in the condition with feedback. Finally, there was no difference in the Pe between the tasks without $(M=12.20, S D=7.11)$ and with feedback $[M=12.91, S D=8.48 ; t(28)=-0.62$, $p>.05]$; however, the Pe was highly correlated between tasks $[r(27)=.71, p<.001]$.

\section{Individual Differences}

The average score on the depression subscale was 9.93 $(S D=9.67)$, with a range of $0-30$. The average score on

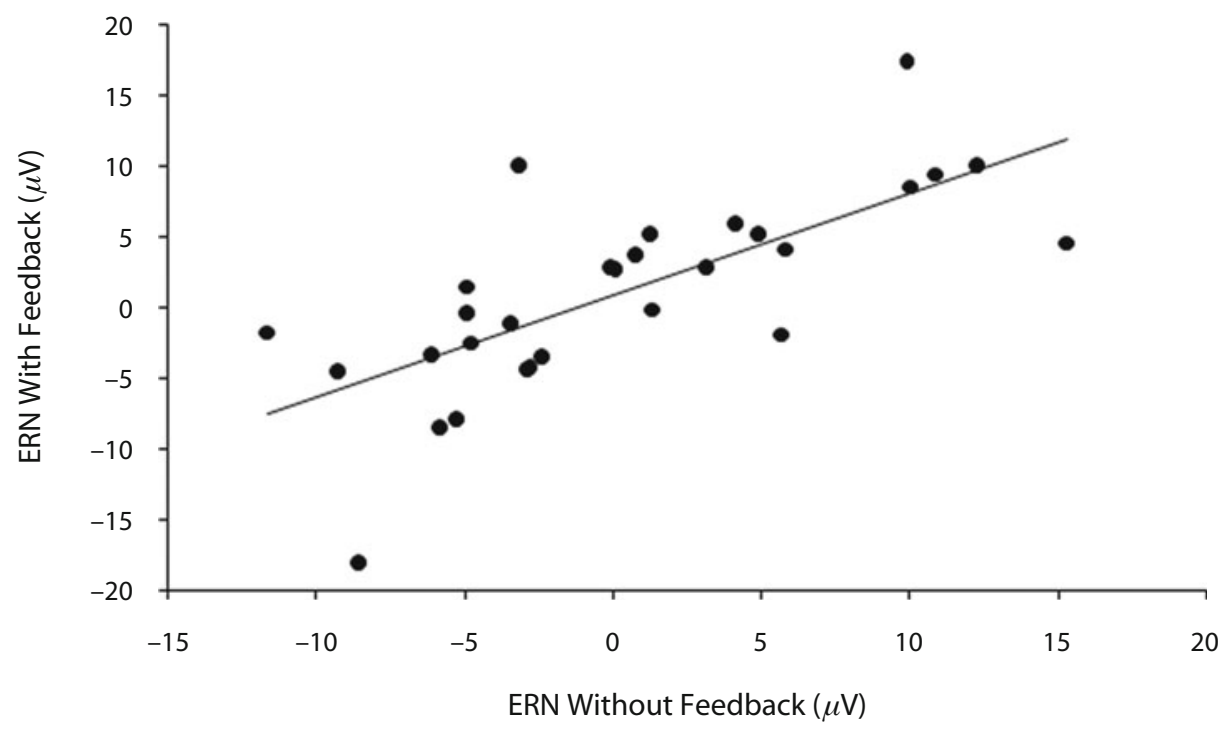

Figure 2. Scatterplot depicting the Pearson correlation for the error-related negativity (ERN) at FCz between the feedback and no-feedback conditions. 
the anxiety subscale was $7.72(S D=8.10)$, with a range of $0-26$. The average score on the stress subscale was 11.31 $(S D=9.60)$, with a range of $0-32$.

The Pearson correlation coefficient was used to explore the relationship between ERPs and self-report measures. There was a significant correlation between the ERN and anxiety in the condition without feedback $[r(27)=$ $-.37, p<.05$; see Figure 3$],{ }^{1}$ indicating that higher anxiety scores predicted a larger ERN; however, the correlation between anxiety and the ERN in the condition with feedback did not reach significance $[r(27)=-.14$, $p>.05]$; the correlations between anxiety and the ERN were significantly different between tasks $(z=4.62, p<$ $.001)$. There were no statistically significant correlations between the ERNs in either condition and the depression or stress scores or between the Pe in either condition and all the self-report measures (all $p \mathrm{~s}>.05$ ).

\section{DISCUSSION}

The present study suggests that the ERN and other error-related components are not directly affected by the presence of trial-to-trial performance feedback. However, trial-to-trial feedback was related to longer reaction times and increased performance accuracy. Together, these findings indicate comparable error-related brain activity despite behavioral differences in the conditions with and without trial-to-trial feedback. In addition, we found that the ERN in the task without feedback was correlated with anxiety scores on the DASS - 21, such that individuals who reported high levels of anxiety also had larger (i.e., more negative) ERNs. This finding was specific to the condition without feedback. Thus, the present results suggest a need to consider the presence of trial-to-trial feedback when the relationship between the ERN and individual differences is examined.
Recently, Gründler et al. (2009) reported smaller ERNs in undergraduates with high OCD symptoms, as compared with those with low OCD symptoms, when a reinforcement learning task was used. This finding was specific to the reinforcement learning task: The participants in the Gründler et al. study also performed a simple flanker task; in this task, those with high OCD symptoms had larger ERNs. Nieuwenhuis et al. (2005) also reported comparable ERNs between individuals with OCD and healthy controls when a reinforcement learning task was used.

On the other hand, most studies to date have shown an increased $\mathrm{ERN}$ in individuals with anxiety disorders, such as OCD (Gehring et al., 2000; Hajcak et al., 2008; Johannes et al., 2001; Ruchsow et al., 2005) and generalized anxiety disorder (Ladouceur, Dahl, Birmaher, Axelson, \& Ryan, 2006), and in individuals reporting anxiety symptoms (Hajcak et al., 2003; Hajcak \& Simons, 2002; Santesso et al., 2006); however, in all of these studies, speeded response tasks without trial-to-trial feedback were used. Our findings suggest that the lack of association between the ERN and anxiety is not specific to reinforcement learning per se but may be due to the presence of trial-to-trial feedback. This possibility is consistent with the suggestion by Nieuwenhuis et al. (2005) that trial-to-trial feedback reduces the encumbrance on response monitoring; that is, trial-to-trial feedback might serve as a kind of reassurance for more anxious individuals and, therefore, alter the relationship between the ERN and trait anxiety.

Although there was no overall difference in the ERN for the tasks with and without feedback, the participants committed fewer errors and were slower to respond when they received trial-to-trial performance feedback. In both conditions in the present study, accuracy and speed were emphasized equally, yet the participants responded more cautiously when given trial-to-trial feedback. Two prominent computational models of the ERN suggest that the

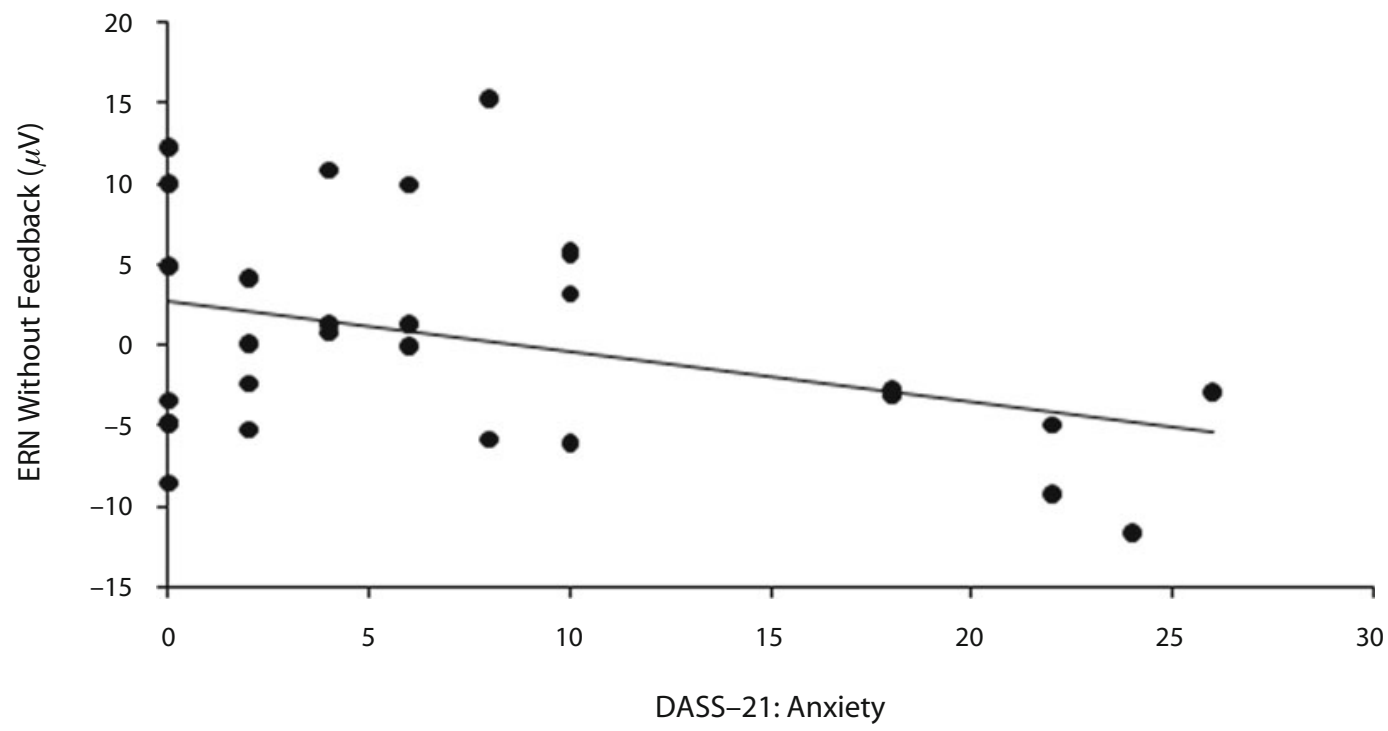

Figure 3. Scatterplot depicting the Pearson correlation between the error-related negativity (ERN) at FCz in the condition without feedback and DASS -21 anxiety scores. 
ERN should be larger when fewer errors are committed (Holroyd \& Coles, 2002; Yeung et al., 2004). Indeed, when accuracy is emphasized over speed, the ERN appears larger in within-subjects designs (Gehring et al., 1993). However, better performance was not characterized by an increased ERN in the present study. Thus, it is possible that behavioral differences alone might not account for ERN differences when accuracy is emphasized over speed (cf. Gehring et al., 1993). Another possibility is that by emphasizing accuracy over speed, the motivational value of errors is increased, and if errors become more motivationally significant, the brain may signal the need for enhanced internal error monitoring, leading to an increased error signal (cf. Hajcak, Moser, Yeung, \& Simons, 2005).

The present data indicate that the presence of trial-totrial feedback moderates the relationship between anxiety and the ERN. Future studies might similarly assess this moderation in other psychiatric disorders. For example, studies that have assessed the ERN in other psychiatric disorders, such as depression and substance abuse, have utilized only speeded response tasks with trial-to-trial feedback (Chiu \& Deldin, 2007; Compton et al., 2008; Franken, van Strien, Franzek, \& van de Wetering, 2007; Holmes \& Pizzagalli, 2008; Schrijvers et al., 2008; Schrijvers et al., 2009). Because behavioral studies indicate that individuals with these psychiatric disorders are especially sensitive to negative feedback (Beats, Sahakian, \& Levy, 1996; Elliott, Sahakian, Herrod, Robbins, \& Paykel, 1997; Elliott, Sahakian, Michael, Paykel, \& Dolan, 1998; Garavan \& Stout, 2005; Steffens, Wagner, Levy, Horn, \& Krishnan, 2001), increased error-related brain activity in depression might be evident only in tasks that present trial-to-trial performance feedback. This would lead to an intriguing set of results: Although both anxiety and depressive disorders might be characterized by increased error-related brain activity, the context in which hyperactive response monitoring is observed may differentiate the disorders.

The present findings underscore the importance of assessing variation in task parameters, especially in regard to relating the ERN both to behavioral measures and to individual-difference variables. Although trial-to-trial feedback did not impact the ERN, it altered behavioral measures substantially; a comparable ERN was observed across conditions that varied greatly in terms of reaction time and error rate. Moreover, the relationship between anxiety and the ERN was moderated by whether or not there was trial-to-trial feedback in the flanker task: Anxiety was related to the ERN only in the task without extraneous performance feedback. These data might help reconcile seemingly contradictory findings on the ERN and anxiety and raise interesting questions about how trial-to-trial feedback might moderate the relationship between neural activity during error monitoring and other individual differences and psychiatric populations.

\section{AUTHOR NOTE}

Correspondence concerning this article may be addressed to G. Hajcak, Department of Psychology, Stony Brook University, Stony Brook, NY 11794-2500 (e-mail: greg.hajcak@stonybrook.edu).

\section{REFERENCES}

Amodio, D. M., Jost, J. T., Master, S. L., \& Yee, C. M. (2007). Neurocognitive correlates of liberalism and conservatism. Nature Neuroscience, 10, 1246-1247.

Antony, M. M., Bieling, P. J., Cox, B. J., Enns, M. W., \& Swinson, R. P. (1998). Psychometric properties of the 42-item and 21-item versions of the Depression Anxiety Stress Scales in clinical groups and a community sample. Psychological Assessment, 10, 176-181.

BAND, G. P., \& KoK, A. (2000). Age effects on response monitoring in a mental-rotation task. Biological Psychology, 51, 201-221.

Beats, B. C., Sahakian, B. J., \& Levy, R. (1996). Cognitive performance in tests sensitive to frontal lobe dysfunction in the elderly depressed. Psychological Medicine, 26, 591-603.

Bernstein, P. S., Scheffers, M. K., \& Coles, M. G. H. (1995). "Where did I go wrong?" A psychophysiological analysis of error detection. Journal of Experimental Psychology: Human Perception \& Performance, 21, 1312-1322.

Brown, T. A., Chorpita, B. F., Korotitsch, W., \& Barlow, D. H. (1997). Psychometric properties of the Depression Anxiety Stress Scales (DASS) in clinical samples. Behaviour Research \& Therapy, 35, 79-89.

ChiU, P. H., \& Deldin, P. J. (2007). Neural evidence for enhanced error detection in major depressive disorder. American Journal of Psychiatry, 164, 608-616.

Clara, I. P., Cox, B. J., \& EnNs, M. W. (2001). Confirmatory factor analysis of the Depression Anxiety Stress Scales in depressed and anxious patients. Journal of Psychopathology \& Behavioral Assessment, 23, 61-67.

Compton, R. J., Lin, M., Vargas, G., Carp, J., Fineman, S. L., \& QUANDT, L. C. (2008). Error detection and posterror behavior in depressed undergraduates. Emotion, 8, 58-67.

Crawford, J. R., \& Henry, J. D. (2003). The Depression Anxiety Stress Scales (DASS): Normative data and latent structure in a large non-clinical sample. British Journal of Clinical Psychology, 42, 111-131.

Davies, P. L., Segalowitz, S. J., \& Gavin, W. J. (2004). Development of response-monitoring ERPs in 7- to 25-year-olds. Developmental Neuropsychology, 25, 355-376.

Dehaene, S., Posner, M. I., \& Tucker, D. M. (1994). Localization of a neural system for error detection and compensation. Psychological Science, 5, 303-305.

Elliott, R., Sahakian, B. J., Herrod, J. J., Robbins, T. W., \& Paykel, E. S. (1997). Abnormal response to negative feedback in unipolar depression: Evidence for a diagnosis specific impairment. Journal of Neurology, Neurosurgery, \& Psychiatry, 63, 74-82.

Elliott, R., Sahakian, B. J., Michael, A., Paykel, E. S., \& Dolan, R. J. (1998). Abnormal neural response to feedback on planning and guessing tasks in patients with unipolar depression. Psychological Medicine, 28, 559-571.

Eriksen, B. A., \& ERIKSEN, C. W. (1974). Effects of noise letters on the identification of a target letter in a nonsearch task. Perception \& Psychophysics, 16, 143-149.

Falkenstein, M., Hohnsbein, J., Hoormann, J., \& Blanke, L. (1991). Effects of crossmodal divided attention on late ERP components: II. Error processing in choice reaction tasks. Electroencephalography \& Clinical Neurophysiology, 78, 447-455.

Franken, I. H., van Strien, J. W., Franzek, E. J., \& van de WeterING, B. J. (2007). Error-processing deficits in patients with cocaine dependence. Biological Psychology, 75, 45-51.

Garavan, H., \& Stout, J. C. (2005). Neurocognitive insights into substance abuse. Trends in Cognitive Sciences, 9, 195-201.

Gehring, W. J., Goss, B., Coles, M. G. H., Meyer, D. E., \& DonCHIN, E. (1993). A neural system for error detection and compensation. Psychological Science, 4, 385-390.

Gehring, W. J., Himle, J., \& Nisenson, L. G. (2000). Action-monitoring dysfunction in obsessive-compulsive disorder. Psychological Science, 11, 1-6.

Gratton, G., Coles, M. G., \& Donchin, E. (1983). A new method for off-line removal of ocular artifact. Electroencephalography \& Clinical Neurophysiology, 55, 468-484.

Gründler, T. O. J., Cavanagh, J. F., Figueroa, C. M., Frank, M. J., \& Allen, J. J. B. (2009). Task-related dissociation in ERN amplitude 
as a function of obsessive-compulsive symptoms. Neuropsychologia, 47, 1978-1987.

HAJCAK, G., \& Foti, D. (2008). Errors are aversive: Defensive motivation and the error-related negativity. Psychological Science, 19, 103-108.

Hajcak, G., Franklin, M. E., Foa, E. B., \& Simons, R. F. (2008). Increased error-related brain activity in pediatric obsessive-compulsive disorder before and after treatment. American Journal of Psychiatry, 165, 116-123.

Hajcak, G., McDonald, N., \& Simons, R. F. (2003). Anxiety and errorrelated brain activity. Biological Psychology, 64, 77-90.

HajCAK, G., McDonald, N., \& Simons, R. F. (2004). Error-related psychophysiology and negative affect. Brain \& Cognition, 56, 189-197.

Hajcak, G., Moser, J. S., Yeung, N., \& Simons, R. F. (2005). On the ERN and the significance of errors. Psychophysiology, 42, 151-160.

HajcaK, G., \& Simons, R. F. (2002). Error-related brain activity in obsessive-compulsive undergraduates. Psychiatry Research, 110, 63-72.

HaJCAK, G., \& Simons, R. F. (2008). Oops! . I did it again: An ERP and behavioral study of double-errors. Brain Cognition, 68, 15-21.

Henry, J. D., \& Crawford, J. R. (2005). The short-form version of the Depression Anxiety Stress Scales (DASS -21): Construct validity and normative data in a large non-clinical sample. British Journal of Clinical Psychology, 44, 227-239.

Holmes, A. J., \& Pizzagalli, D. A. (2008). Spatiotemporal dynamics of error processing dysfunctions in major depressive disorder. $\mathrm{Ar}$ chives of General Psychiatry, 65, 179-188.

Holroyd, C. B., \& Coles, M. G. (2002). The neural basis of human error processing: Reinforcement learning, dopamine, and the errorrelated negativity. Psychological Review, 109, 679-709.

Holroyd, C. B., Dien, J., \& Coles, M. G. (1998). Error-related scalp potentials elicited by hand and foot movements: Evidence for an output-independent error-processing system in humans. Neuroscience Letters, 242, 65-68.

Johannes, S., Wieringa, B. M., Nager, W., Rada, D., Dengler, R., EMrich, H. M., ET AL. (2001). Discrepant target detection and action monitoring in obsessive-compulsive disorder. Psychiatry Research, 108, 101-110.

Ladouceur, C. D., Dahl, R. E., Birmaher, B., Axelson, D. A., \& RYAN, N. D. (2006). Increased error-related negativity (ERN) in childhood anxiety disorders: ERP and source localization. Journal of Child Psychology \& Psychiatry, 47, 1073-1082.

Lovibond, P. F., \& LoviBond, S. H. (1995). The structure of negative emotional states: Comparison of the Depression Anxiety Stress Scales (DASS) with the Beck Depression and Anxiety Inventories. Behaviour Research \& Therapy, 33, 335-343.

Luu, P., Collins, P., \& Tucker, D. M. (2000). Mood, personality, and self-monitoring: Negative affect and emotionality in relation to frontal lobe mechanisms of error monitoring. Journal of experimental psychology: General, 129, 43-60.

Mathalon, D. H., Bennett, A., Askari, N., Gray, E. M., RosenBLOOM, M. J., \& FORD, J. M. (2003). Response-monitoring dysfunction in aging and Alzheimer's disease: An event-related potential study. Neurobiology of Aging, 24, 675-685.

Moser, J. S., HajcaK, G., \& Simons, R. F. (2005). The effects of fear on performance monitoring and attentional allocation. Psychophysiology, 42, 261-268.

Nieuwenhuis, S., Nielen, M. M., Mol, N., Hajcak, G., \& Veltman, D. J. (2005). Performance monitoring in obsessive-compulsive disorder. Psychiatry Research, 134, 111-122.

Nieuwenhuis, S., Ridderinkhof, K. R., Talsma, D., Coles, M. G. H.,
Holroyd, C. B., Kok, A., \& van der Molen, M. W. (2002). A computational account of altered error processing in older age: Dopamine and the error-related negativity. Cognitive, Affective, \& Behavioral Neuroscience, 2, 19-36.

Olvet, D. M., \& HAJCAK, G. (2008). The error-related negativity (ERN) and psychopathology: Toward an endophenotype. Clinical Psychology Review, 28, 1343-1354.

Olvet, D. M., \& HaJcaK, G. (2009). The stability of error-related brain activity with increasing trials. Psychophysiology, 46, 957-961.

Rabbitt, P., \& Vyas, S. (1981). Processing a display even after you make a response to it: How perceptual errors can be corrected. Quarterly Journal of Experimental Psychology, 33A, 223-239.

Ridderinkhof, K. R., Ullsperger, M., Crone, E. A., \& NieuwenHuIS, S. (2004). The role of the medial frontal cortex in cognitive control. Science, 306, 443-447.

Ruchsow, M., Gron, G., Reuter, K., Spitzer, M., Hermle, L., \& Kiefer, M. (2005). Error-related brain activity in patients with obsessive-compulsive disorder and in healthy controls. Journal of Psychophysiology, 19, 298-304.

Santesso, D. L., Segalowitz, S. J., \& Schmidt, L. A. (2006). Errorrelated electrocortical responses are enhanced in children with obsessive-compulsive behaviors. Developmental Neuropsychology, 29, 431-445.

Schrijvers, D., De Bruijn, E. R. A., MaAs, Y. [J.], De Grave, C., Sabbe, B. G. C., \& Hulstiun, W. (2008). Action monitoring in major depressive disorder with psychomotor retardation. Cortex, 44, 569-579.

Schrijvers, D., De Bruijn, E. R. A., MaAs, Y. J., Vancoillie, P., HulSTIJN, W., \& SABbE, B. G. C. (2009). Action monitoring and depressive symptom reduction in major depressive disorder. International Journal of Psychophysiology, 71, 218-224.

Steffens, D. C., Wagner, H. R., Levy, R. M., Horn, K. A., \& KrishNAN, K. R. (2001). Performance feedback deficit in geriatric depression. Biological Psychiatry, 50, 358-363.

Themanson, J. R., Hillman, C. H., \& Curtin, J. J. (2006). Age and physical activity influences on action monitoring during task switching. Neurobiology of Aging, 27, 1335-1345.

VAN'T ENT, D., \& APKARIAN, P. (1999). Motoric response inhibition in finger movement and saccadic eye movement: A comparative study. Clinical Neurophysiology, 110, 1058-1072.

VAN VEEN, V., \& CARTER, C. S. (2002). The timing of action-monitoring processes in the anterior cingulate cortex. Journal of Cognitive Neuroscience, 14, 593-602.

Yeung, N., Cohen, J. D., \& Botvinick, M. M. (2004). The neural basis of error detection: Conflict monitoring and the error-related negativity. Psychological Review, 111, 931-959.

\section{NOTE}

1. Anxiety and depression were highly correlated in the present sample $(r=.76, p<.001)$. To explore the specificity of the relationship between the ERN in the condition without feedback and anxiety, we performed a partial correlation between the ERN and anxiety, including depression as a covariate. The results were in the same direction; however, the correlation did not reach significance $(r=-.32, p=.1)$. The correlation between the ERN in the condition with feedback and anxiety was still nonsignificant when depression was included as a covariate $(r=-.00, p>.05)$.

(Manuscript received March 2, 2009; revision accepted for publication August 5, 2009.) 